\title{
3D droplet displacement in microfluidic systems by electrostatic actuation
}

\author{
Jean-Maxime Roux ${ }^{\mathrm{a}, *}$, Yves Fouillet ${ }^{\mathrm{a}}$, Jean-Luc Achard ${ }^{\mathrm{b}}$ \\ ${ }^{a}$ CEA/LETI - Laboratoire d'Electronique et de Technologie de l'Information, 17 av. des Martyrs, 38054 Grenoble Cedex 9, France \\ ${ }^{\mathrm{b}}$ Laboratoire des Ecoulements Géophysiques et Industriels, 38041 Grenoble Cedex 9, France
}

\begin{abstract}
A conducting droplet set on the lower plate of a condenser acquires an electrical charge when subjected to an electrical field. Above a threshold value the electrical force lifts the droplet. If the droplet's charge does not leak away, the droplet moves up to the upper electrode and bounces on it. Coating the upper electrode with a dielectric layer allows the otherwise regular droplet back and forth motion to be controlled. This new idea is applied to a droplet-based microfluidic system which drives droplets by electrowetting on a planar array. In this way additional flexibility is achieved since droplets can be displaced and controlled in the three space dimensions.
\end{abstract}

Keywords: Microfluidics; Droplet displacement; Electrohydrodynamic

\section{Introduction}

Droplet or "digital" microfluidics enables a whole range of operations (droplet dispensing, displacement, coalescence, etc.) affecting droplets, which act as genuine microreactors, for integrating chemical or biological protocols [1]. Our work concerns the study of the electrostatic forces exerted on the fluid/fluid interfaces to perform more flexible droplet displacements. A fundamental study, both experimental and theoretical, was made on the behavior of a water droplet into a plate condenser whose dielectric is a liquid immiscible with the droplet. The goal is to add a vertical motion to a standard electrowetting [2] device on which micro-droplets can be displaced along a single horizontal surface. More precisely, the aim is to bring, in a controlled manner, the droplet from the lower first single plane to a higher plane [3], by the action of electrostatic forces, without the droplet remaining stuck on this higher plane. The resulting microfluidic system is flexible. It enables the uncoupled use of the three spatial dimensions: the 2D space of the lower substrate is devoted to the displacement of droplets, while the higher substrate, via vertical displacement on certain previously determined sites, storage zones or functionalized sites, provides progressive completion of chemical and biological

\footnotetext{
* Corresponding author. Tel.: +33 438782230; fax: +33 438785787

E-mail address: rouxjm@chartreuse.cea.fr (J.-M. Roux).
}

operations. Many applications can be foreseen. In particular, it is possible to reduce significantly the time required to complete certain classic molecular biology protocols. For example, the micro-droplet containing all the reagents required for DNA amplification could be transferred very quickly from one heating site to another: the thermal cycle time would be considerably reduced.

First, we present the possibility of lifting a water microdroplet, in a liquid dielectric, from one plate of a plane condenser. We then show that depending on the configurations used, the movement of the micro-droplet can be either an uncontrolled back and forth movement between the condenser electrodes [4] or, on the contrary, as in our case, a controlled transfer of the droplet from one stable position on one plate to another stable position on the other plate. Theoretical elements borrowed from Lebedev and Skal'skaya [5] and Jones [6] are introduced to interpret the droplet behavior.

The final part describes the development of an innovative microfluidic system that associates the studied displacements and electrowetting-based displacements along a flat surface. We demonstrate that the droplets formed in the micro-device from the on-chip reservoirs can be displaced either by electrowetting on a flat surface of the device or fly from the electrowettingbased chip surface to reach another plate; all operations being reversible. The proposed device therefore broadens the possibilities of electrowetting and introduces a new generation of microfluidic systems enabling complex droplet manipulation. 


\section{Droplet take-off}

A fundamental starting point is provided by an old theoretical study of Lebedev and Skal'skaya [5]. These authors determined the charge $Q_{0}$ (1) taken by a perfectly conductive sphere, of radius $R$, in contact on one of the plates of a plate condenser and subjected to the electric field $\mathbf{E}$ between the two plates. In this configuration, the resulting electrostatic force (2) exerted on the sphere perpendicularly to these plates was also calculated. One restriction is that the radius $R$ of this sphere must be negligible compared to the inter-plate distance $h$ :

$Q_{0}=4 \pi \varepsilon \varepsilon_{0} E R^{2} \zeta(2)$

$F_{z}=4 \pi \varepsilon \varepsilon_{0} E^{2} R^{2}\left\{\zeta(3)+\frac{1}{6}\right\}$

The function $\zeta$ is Riemann's Zeta function and $E=|\mathbf{E}|$.

Under the effect of an electric field, the free charge carriers of a perfect conductor are spread over its surface; global charge and electric force must therefore be linked to the conductor surface. In expressions (1) and (2), these values are proportional to the sphere's surface area.

It has been demonstrated that the acquired charge $Q_{0}$, called the Maxwell charge, depends on the sign of the electric field, but the force $F_{z}$ exerted along the axis perpendicular to the electrode always tends to detach the sphere. Indeed the force being proportional to the square of the applied electric field intensity, it is independent of the field sign. Whatever the sign of the electric field the acquired charges force the sphere to depart from the electrode. However the study of Lebedev and Skal'skaya [5] does not explain the exact nature of the electrostatic force $F_{z}$ which is not only the Coulombian force $Q_{0} E$.

The previous approach, based on the use of bispherical coordinates was taken up and confirmed later by Jones [6] using a multipolar approach. Here, the force is divided into one component tending to lift the sphere (Coulombian force $Q_{0} E$ relative to the global charge taken by the sphere) and one attractive component, due to the mutual force of attraction between the sphere and its image in relation to the plate; indeed, the presence of the electrode with uniform potential imposes deformation of the electric field around the sphere. More recently, Takahashi et al. [7] has studied the motion of a micro-ball into a plate condenser and numerically calculated (boundary integral method) the departing force. In their study $R / h$ is not necessarily small compared to 1. However, the exact results (Eqs. (1) and (2)) of Lebedev and Skal'skaya [5], apparently unknown by Takahashi, seem difficult to repeat when $R / h$ tends towards 0 . The experimental work of Saito et al. [8] confirms this numerical work.

To determine a sphere take-off criterion, the electrostatic force Eq. (2) must be equal to the sum of resisting forces exerted at take-off. Two situations are examined below: a solid sphere and, finally, a droplet which is of interest in this paper.

A solid sphere heavier than the surrounding fluid is deposited on a horizontal electrode. Above a certain inter-plate voltage, the electrostatic force will cause the sphere to take-off. The threshold voltage for lifting a sphere can be evaluated by writing that the electrostatic force Eq. (2) determined by Lebedev and

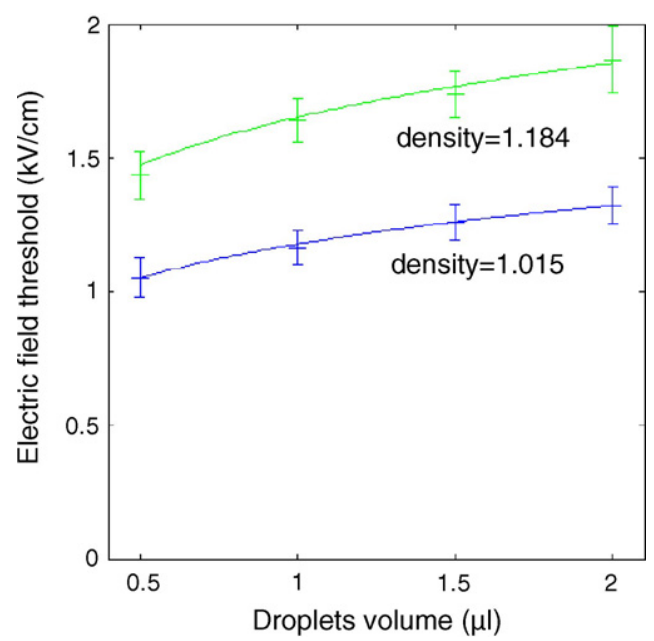

Fig. 1. Experimental and theoretical take-off field threshold plotted as functions of the droplet volume. The solid line is the theoretical threshold.

Skal'skaya [5] must at least equal the weight of the sphere minus buoyancy.

We have transposed the situation of solid spheres placed on an electrode to droplets by using a conducting superhydrophobic [9] lower electrode. Hydrophobic carbon nanotubes covered electrodes are so hydrophobic that droplets behave as liquid balls on them. In our experiments, water droplets from two different $\mathrm{NaCl}$ solutions (a $0.5 \mathrm{M}$ solution of density 1.015 and a saturate solution of density 1.184) were introduced in a plate condenser filled with a dielectric mineral oil of density 0.84 and relative permittivity 2.11 . In Fig. 1, the minimum electrostatic field to lift droplets is plotted as a function of droplets volume. The theoretical prediction shows an excellent agreement with the experimental voltage threshold. So the theory, valid for solid conducting balls, can be extended to conducting droplets.

If the lower electrode is not superhydrophobic [4] the electrical force must overcome not only the weight of the droplet minus buoyancy but also the capillary force $2 \pi \delta \gamma \sin (\theta)$, where $\delta$ is the radius of contact area, $\theta$ the contact angle and $\gamma$ is the surface tension of the droplet. The driving force, the weight and buoyancy expressions are known but the capillary force is a complex function of the electrical field and droplet shape. To our knowledge, the problem has not been solved yet. Furthermore, the wetting properties which are often difficult to control and to reproduce, lead to high uncertainty on the threshold. In Section 4 we will use electrodes with varying wetting properties. To avoid any uncertainty to take-off droplets we will impose electrical field values which exceed the theoretical threshold values with a safety margin of $30 \%$.

\section{Back and forth displacement between two bare electrodes}

When a step voltage is applied between the condenser electrodes, such a droplet is subject to the action of upward force Eq. (2) that opposes the capillary force and the weight minus buoyancy. For a constant electric field $\mathbf{E}$ of sufficient intensity the microliter droplet, leaves the electrode on which it was placed 
and moves towards the condenser's upper electrode. After contact with the bare upper electrode, it immediately moves back down. The droplet takes a regular forced back and forth movement between the two plates of the condenser [4]. In general, there is no stable position as long as the global electric force exceeds the weight.

\subsection{Physical considerations}

After being lifted from the lower electrode, the droplet moves towards the upper electrode and bounces against it; this contact brings the droplet back to an electrical state identical to that on the lower electrode before take-off. Indeed the droplet acquires the opposite sign charge and becomes subjected to the force (2) evaluated by Lebedev and Skal'skaya [5], but downwards this time.

After departing from one electrode, the charge acquired by a droplet leaks away from interface for times longer than the relaxation time. Assuming an ohmic regime for charge transfers, this relaxation time $\tau$ defined in (4) appears in the conservation equation (3); this is the time required for the charges to relax in the liquid dielectric and restore electric equilibrium:

$\frac{\partial q}{\partial t}+\frac{\sigma}{\varepsilon} q=0$

$\tau=\frac{\varepsilon}{\sigma}$

For the mineral oil used in our experiments, relative permittivity and conductivity are 2.11 and $(396 \pm 4) \times 10^{-15} \mathrm{~S} \mathrm{~m}^{-1}$, giving $\tau=47 \mathrm{~s}$. For times much smaller than $\tau$, free charges carriers are still located on the liquid/liquid interface and the dielectric can be considered as perfect. The typical droplet transit time between the two electrodes being about $200 \mathrm{~ms}$ in our case, it is possible to disregard the charges leaking process between two impacts. As a consequence, during a flight from one electrode to the other, the droplet charge $Q$ is assumed to be the charge taken at the last impact. $Q$ was shown to be different [11] from the Maxwell charge $Q_{0}$ when taken during an impact because charges are transferred at a small distance from the electrode. However, the Maxwell charge defined in Eq. (1) gives the order of magnitude of $Q$ so below it will be assumed that $Q=Q_{0}$.

A droplet of radius $R$, negligible compared to inter-plate distance $h$, can therefore be assimilated with a conductive sphere bearing a fixed charge $Q_{0}$, near a single conductive flat surface and subject to electric field $\mathbf{E}$. Thanks to the theorem of images this problem can be compared with that studied by Davis [10] of two charged spheres bearing charges $Q_{0}$ and $-Q_{0}$ placed in a uniform electric field E (Fig. 2). Davis [10] calculated the spheres potentials and the forces exerted on them. By imposing a constant charge $Q_{0}$ for calculations, we checked numerically that the force $F_{z}$ exerted on a sphere increases with the distance, from the force calculated by Lebedev and Skal'skaya [5] at $z=0$ to the Coulombian force $Q_{0} E$ at infinity. Fig. 3 shows the evolution of the two components of the electric force on a sphere; the curves have been normalized with $Q_{0} E$. The figure shows that the influence of the electrode for the sphere disappears after $z / R=4$.

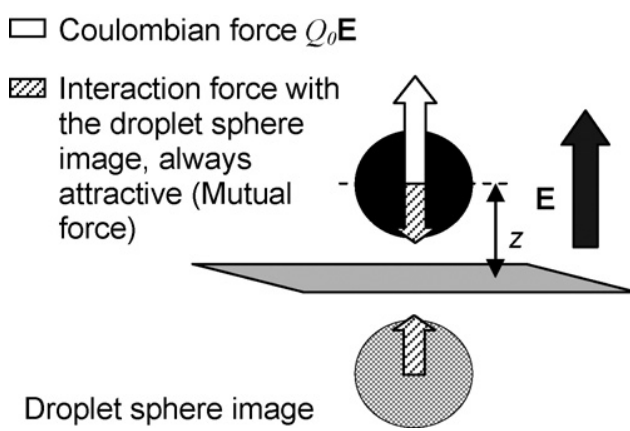

Fig. 2. Electrical forces exerted on a conducting sphere near a conductive plane.

The distance at which the force reaches the plateau defined by $Q_{0} E$ is the distance at which the electrode stops exerting an influence; the electric force is then reduced to the action of the uniform electric field on the overall charge carried by the droplet from its departure from the electrode. This result provides also an estimate of the error made in neglecting the influence of the second electrode of our device, depending on distance $h$.

Our aim here is to calculate the order of magnitude of the droplet transit time. A droplet of volume $V=4 / 3 \pi R^{3}$ and density $\rho_{i}$ is subject to electrostatic and hydrodynamic forces after takeoff. The main driving force is an electrical one. Since leaking effects can be neglected this force is mainly the Coulombian force $Q_{0} E$.

The Coulombian force must be balanced by resisting forces. A first resisting force is the weight of the droplet minus buoyancy: $\left(\rho_{i}-\rho_{e}\right) V g$. Another resisting force is inertia $\rho_{i} V \mathrm{~d}^{2} z / \mathrm{d} t^{2}$, where $z$ is the height of the droplet centre measured from the lower electrode. The added mass force (due to fluid inertia) far from the plates is $(1 / 2) \rho_{e} V \mathrm{~d}^{2} z / \mathrm{d} t^{2}, \rho_{e}$ being the oil density. This force has the same order of magnitude as inertia since densities are similar $\left(\rho_{i}=10^{3} \mathrm{~kg} / \mathrm{m}^{3}\right.$ and $\left.\rho_{e}=0.84 \times 10^{3} \mathrm{~kg} / \mathrm{m}^{3}\right)$. Finally the last resisting force is the viscous drag $4 \pi \mu_{e} R \mathrm{~d} z / \mathrm{d} t$ ( $\mu$ being the dynamic viscosity) which is given by the limiting form of the Hadamard-Rybczynski law for $\mu_{i} / \mu_{e} \ll 1$. This force will be considered as the main resisting force. The momentum equation can be non-dimensionalized by introducing $\tau_{h}$ as the time scale

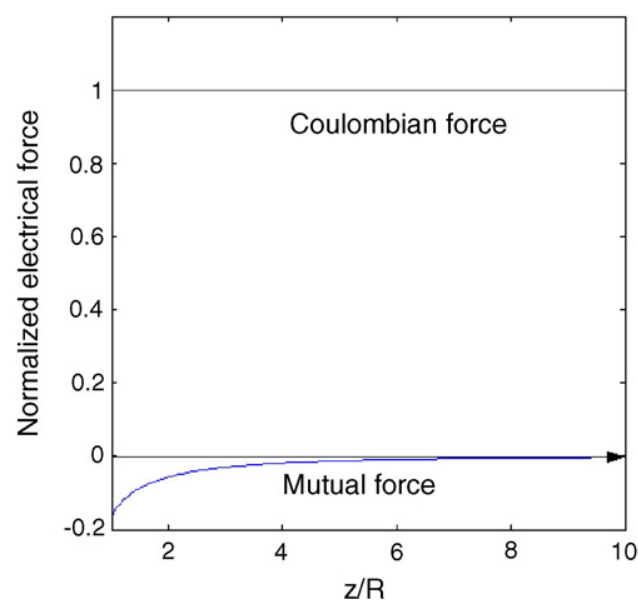

Fig. 3. Conductive sphere subjected to a uniform electric field near a flat electrode. Evolution of the two components of the force is indicated. 
corresponding to the droplet transit time ( $\sim 200 \mathrm{~ms})$ between the two electrodes separated by a distance $h$. The equilibrium of the main resistive and driving forces described above naturally leads to the estimation of $\tau_{h}$ :

$\tau_{h}=\frac{3 h / \tau_{v}}{\left(Q_{0}|\mathbf{E}| / \rho_{e} V\right)+\left(1-\left(\rho_{i} / \rho_{e}\right)\right) g}$

where $\tau_{v}=R^{2} v_{e}$ is the viscous time scale, $10 \mathrm{~ms}$ in our experiments.

For a $2 \mathrm{kV} \mathrm{cm}^{-1}$ electric field, a $1 \mu \mathrm{l}$ water droplet should travel a distance of $7.8 \mathrm{~mm}$ in $\tau_{h}=441 \mathrm{~ms}$. Another assumption would correspond to an equilibrium between the resisting inertia term and the driving force. Such hypothesis would have provided a smaller value of $\tau_{h}$, say $50 \mathrm{~ms}$. Both estimations are not quite satisfactory showing that both resisting forces are present in our experiments. However our initial choice based on the viscous drag seems better and furthermore is especially justified for smaller droplets which can be of interest for further applications. Their inertia will be indeed less and less important.

In comparison, Tobazéon [11] in experiments where the viscous time scale $\tau_{v}$ was $25 \mathrm{~ms}$ reported velocities of $0.2-1 \mathrm{~m} / \mathrm{s}$ for steel spheres in insulating oil subjected to an electrical field of $8-30 \mathrm{kV} / \mathrm{cm}$. Based on an average Reynolds number of 9-90 he concluded that the hydrodynamic regime was an intermediate regime between viscous and inertial.

\section{Back and forth displacement control}

\subsection{Experimental set-up}

The condenser plates, Fig. 4, are circular, made of aluminum and separated by $10 \mathrm{~mm}$ height spacers. These electrodes are polarized by a TreK 677B high voltage generator controlled by a Yokogawa FG120 function generator. Droplets of about $1 \mu \mathrm{l}$ are introduced using a capillary of $105 \mu \mathrm{m}$ outer diameter and $40 \mu \mathrm{m}$ inner diameter. The condenser is immersed in a tank filled with light mineral oil (M-3516 Sigma, density 0.84 and kinematic viscosity $\left.v_{e}=36 \times 10^{-2} \mathrm{~cm}^{2} / \mathrm{s}\right)$. Observations are made with a KAPPA CD11DSP video camera, fitted with a Computar Macro $10 \times$ lens, through the glass wall of the tank. Video acquisition and image extraction operations are performed using Adobe Premiere 6.5 software.

In the following experiments a $1 \mathrm{M} \mathrm{NaCl}$ solution $(12 \Omega \mathrm{cm})$ is used to create very conducting droplets. A surfactant (Tween $20,1 \%$ volume) was added to the solution in order to form droplets of $1 \mu \mathrm{l}$ with the capillary. An average $200 \mathrm{~ms}$ transit time was measured from the video acquisition for droplets of $1 \mu \mathrm{l}$ subjected to a $2 \mathrm{kV} / \mathrm{cm}$ field on a $7.8 \mathrm{~mm}$ displacement.

\subsection{Control}

One way of stopping the back and forth movement of the droplet due to charge transfers on both electrodes is to prevent electric charge transfers on the upper electrode using a solid dielectric, preferably hydrophobic (Fig. 4). In the experiments presented, the upper electrode is insulated by a $1 \mathrm{~mm}$

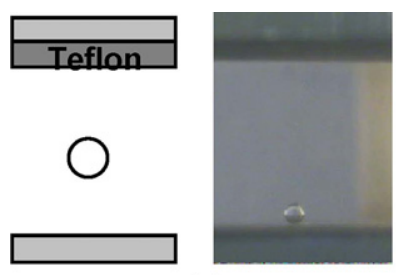

(a)

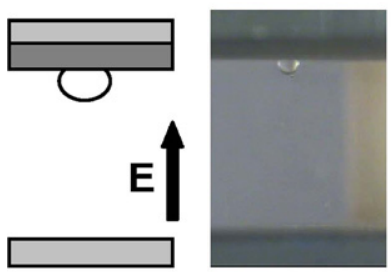

(c)

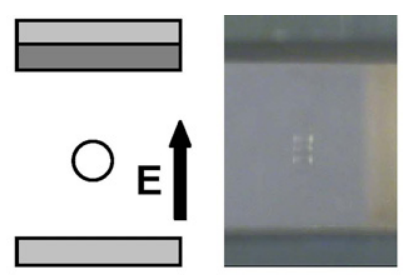

(b)

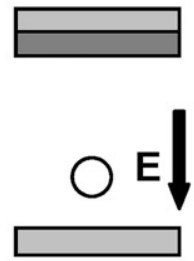

(d)
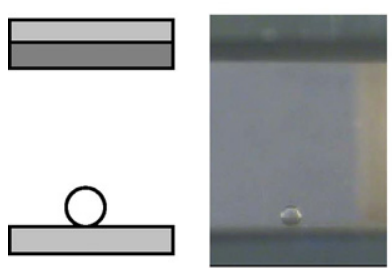

(e)

Fig. 4. Experimental study of a $1 \mu l$ water droplet displacement between the $10 \mathrm{~mm}$ spaced plates of a plate condenser. The Teflon surface insulating the upper electrode is $1 \mathrm{~mm}$ thick. The voltage sequence is as follows: $0,+1600$, -1600 , and $0 \mathrm{~V}$. (a) The droplet rests on the lower electrode. (b) The imposed voltage causes the droplet to move towards the upper electrode. (c) The electric force maintains the droplet against the dielectric layer. (d) By inverting the sign of the imposed voltage, the Coulombian force exerted on the global charge of the droplet is inverted and the droplet moves back down. (e) A moment before contact between the droplet and the lower electrode, zero voltage is imposed to the condenser. The droplet rests on the lower electrode.

thick Teflon surface. When it reaches the dielectric layer, the droplet is crushed against it (Fig. 1c) by the electric force that cannot change sign because the charge cannot be transferred to the upper electrode.

By inverting the electrical field, the electrical force exerted on the droplet can be reversed. Furthermore, because the dielectric used is hydrophobic (wetting forces are small), the droplet can be moved back down by inverting the sign of the electric field. To maintain the droplet on the lower electrode, the electric voltage imposed to the condenser must be less than the take-off voltage threshold.

As the droplets can be retained by capillary forces in one position, it appears useful to use hydrophobic surfaces. However, the wetting properties of the lower electrode are less essential than that of the insulated upper electrode. Indeed, at the top, the electric constraint forces wetting; at the bottom, the effect of the weight, compensated by buoyancy, is comparatively weak and electrode wetting can take longer. In order to be within the theoretical models hypothesis, the electrodes must be perfectly conductive with direct contact with the droplet and minimum wetting surface area. These constraints (conductive and hydrophobic) are often antagonistic and depending on the applications, either one or the other may be favored. In our exper- 
iments, the metal surface of the lower electrode was left bare. However, in a demonstrator, since the resting time of a droplet is imposed by the application and may be long, all surfaces will be hydrophobic.

\section{Development of a fluidic demonstrator}

For lab-on-chip applications, several physical principles have been suggested to handle droplets on an $X-Y$ plane: dielectrophoresis (DEP) [12-14], thermocapillarity [15], acoustic waves [16] and electrowetting. To our knowledge, none of these applications uses $Z$ displacements. To a certain extent, one may observe that dielectrophoresis-based fluid processors are concerned about this vertical dimension. Droplets which are manipulated through the DEP force do not require surface contact and wetting is even avoided for contamination issues. But droplets are just suspended by the electrical force at some constant level above insulated electrodes. Note that Velev et al. [14] used buoyancy forces instead of the vertical component of the DEP force to keep droplets floating above the electrodes array.

Developments have demonstrated that amongst the different principles, electrowetting on dielectric (EWOD), which modifies the 'apparent' wettability by electric actuation [17], enables a wide range of basic fluidic operations required to analyze biological [18] or chemical samples. Creating, transporting, cutting and merging liquid droplets $[19,20]$ containing samples and reagents can be performed on the same chip by programming an electrode array rather than adding up physical structures for accomplishing these fluidic functions. EWOD based devices are very promising because the chip manufacturing process is much simpler, and there is no need for moving micromechanical parts or external pumping systems.

EWOD devices are divided into two categories [1]: closed systems in which droplets are confined between two parallel plates and open systems in which droplets lie on the chip surface (Fig. 5).

The fundamental work presented above has led to the design of a new hybrid EWOD/vertical displacement-based device

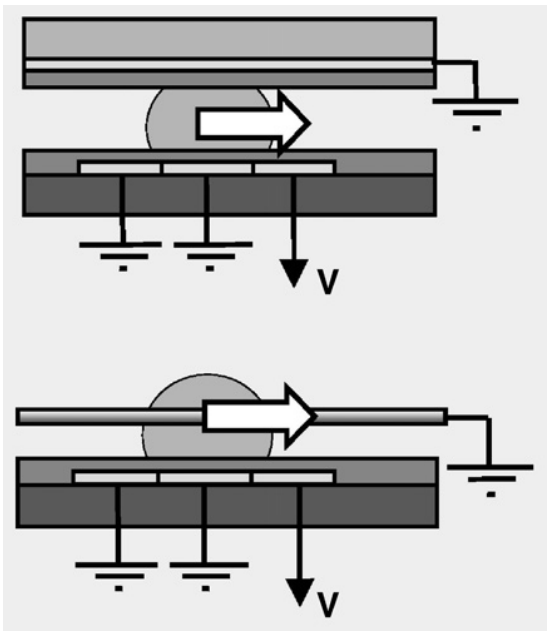

Fig. 5. The different classes of EWOD based systems: closed systems and open systems.

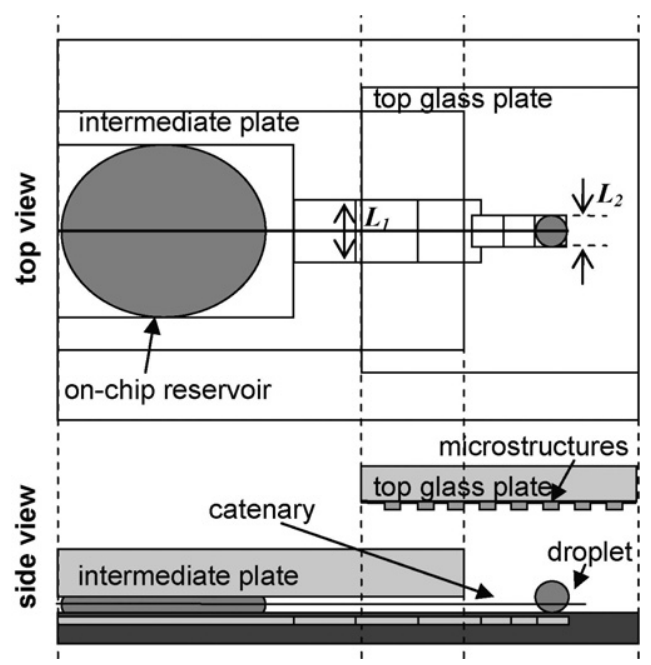

Fig. 6. Schematic description of the device: top and side view.

whose schematic is shown in Fig. 6. The electrowetting system consists, firstly, of a standard closed electrowetting system in which droplets may be dispensed from an on-chip reservoir and, secondly, of an open electrowetting system where the top plate is kept at a distant position from the lower plate. Our work consists precisely to transfer droplets between these two plates in a controlled way. Furthermore, the open system lower plate consists of an EWOD based chip on which the fluidic operations on droplets are performed and a functionalized top plate on which biological or chemical analyses are proceeded.

EWOD technology is usually based on collective and well known micro-technology processes involving successive deposition, photolithography and etching steps. A $3000 \AA$ A thick layer of gold is deposited on an insulating substrate such as glass or silicon oxide. The gold electrodes are etched and covered with a dielectric layer of silicon nitride $3000 \AA$ thick. A gold microcatenary $(25 \mu \mathrm{m})[2,21,22]$ in contact with droplets is added by wire bonding; it forms the ground electrode for the EWOD based operations and the vertical displacement described in the previous paragraphs. The top glass plate is coated with a continuous $1400 \AA$ A thick layer of optically transparent indium tin oxide (ITO) to form the upper electrode, insulated by a $3000 \AA$ thick dielectric layer of silicon nitride and patterned by a $100 \mu \mathrm{m}$ thick dry film (Ordyl). Surfaces are made hydrophobic by depositing a thin layer of Teflon-like substance of a few hundred nanometers.

The silicon chip is directly plugged into a standard card socket from SAMTEC with no bonding operation.

In the experiments reported in this paper, electrodes are $1.6 \mathrm{~mm}$ large $\left(L_{1}\right)$ in the closed EWOD zone and $0.8 \mathrm{~mm}$ large $\left(L_{2}\right)$ in the open EWOD zone. The intermediate plate made of $1 \mathrm{~mm}$ polycarbonate was separated from the chip by $355 \mu \mathrm{m}$ spacers and the upper plate was separated from the chip by a $2 \mathrm{~mm}$ multilayer polycarbonate frame. Cylinders of Ordyl $400 \mu \mathrm{m}$ wide, $100 \mu \mathrm{m}$ high and located $100 \mu \mathrm{m}$ apart from each other were made on the top glass plate in order to prevent passively the droplets from sliding. On-chip reservoirs were filled with deionized water or TBE with fluorescein (from Molecular 
(a)

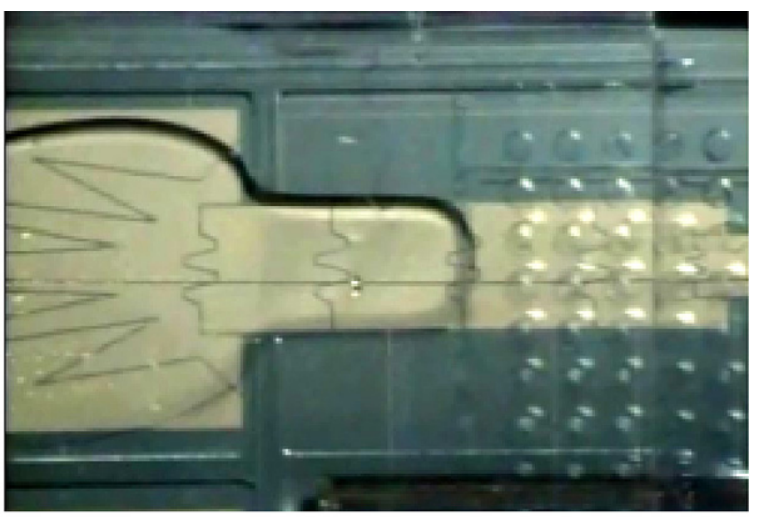

(b)

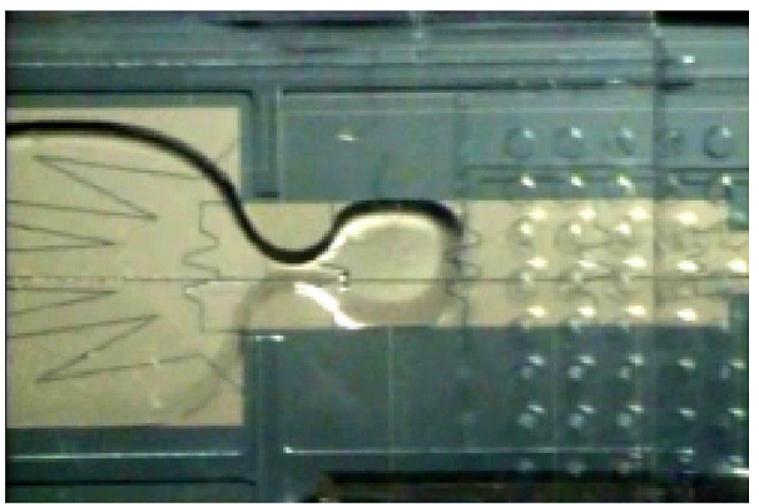

(c)

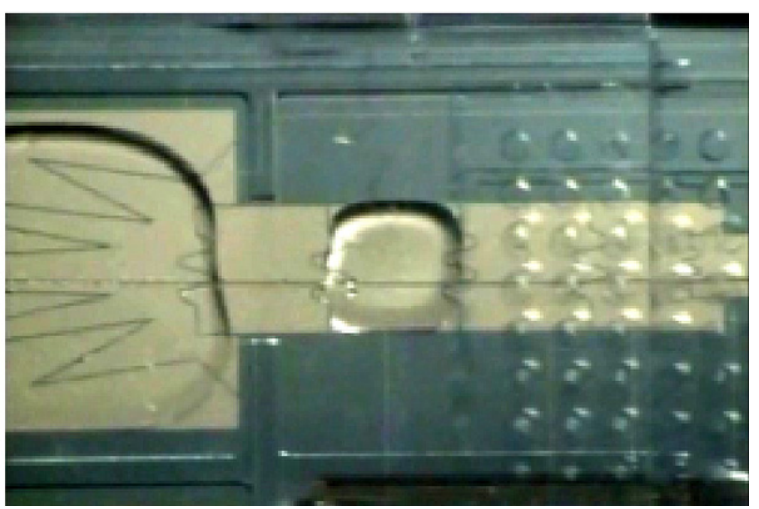

Fig. 7. Droplets are dispensed from an on-chip reservoir onto $1600 \mu \mathrm{m}$ wide electrodes.

Probes) $100 \mu \mathrm{M}$. Light mineral oil from Brookfield (fluid 10: viscosity $9.5 \mathrm{cP}$ and density 0.98 at $20^{\circ} \mathrm{C}$ ) was used as the filler dielectric medium in all experiments to prevent evaporation and reduce the weight of the droplets to be lifted.

\subsection{Droplet dispensing}

On-chip dispensing was accomplished by first extending a liquid column (Fig. 7a) from an on-chip reservoir by activating a series of electrodes $[19,20]$. The electrode in the reservoir was activated to retract the liquid and pinch-off a droplet over a deactivated electrode (Fig. 7b). $0.25 \mu \mathrm{l}$ and $0.92 \mu \mathrm{l}$ water droplets were successfully dispensed from an on-chip reservoir (Fig. 7c) on $1600 \mu \mathrm{m}$ wide electrodes with spacers of 100 and $360 \mu \mathrm{m}$ height, respectively (Fig. 7).
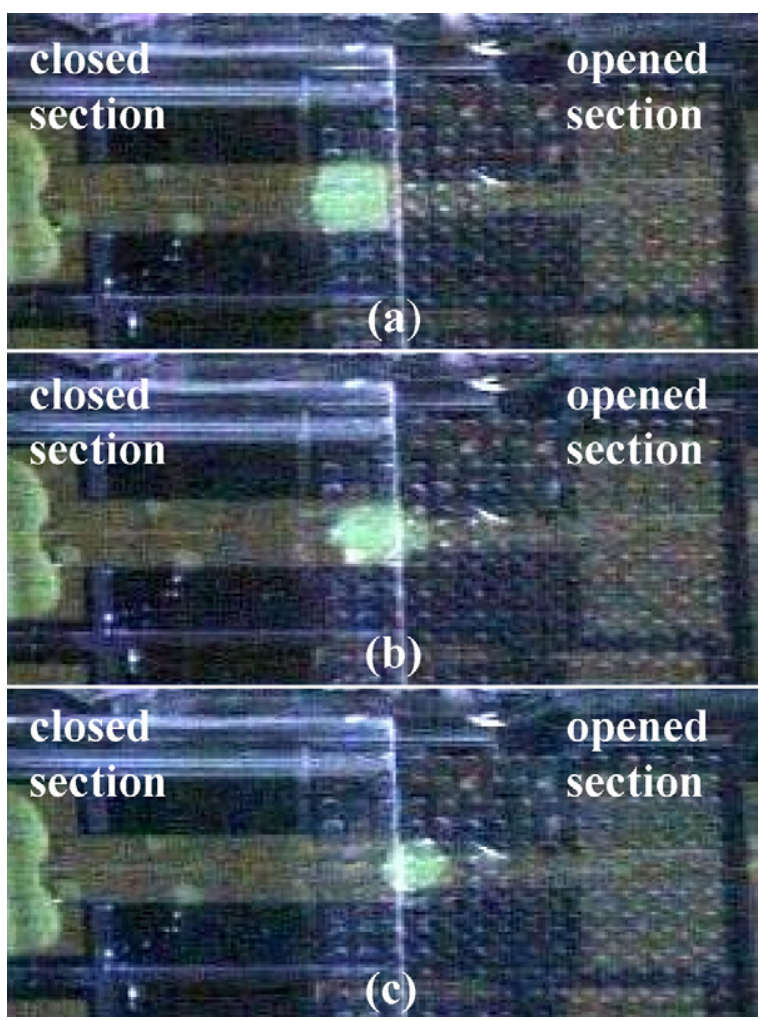

Fig. 8. From the closed EWOD configuration (a) to the open one (c). A droplet of fluorescein was dispensed in the closed chip and moved to the open one; spacers are $360 \mu \mathrm{m}$ high.

Dispensed droplets are moved from the closed EWOD system to the open one (Fig. 8) and eventually back. Both 100 and $360 \mu \mathrm{m}$ spacers were tested but only the highest spacers enable a back and forth movement from the closed to the opened EWOD system for deionized water droplets.

\subsection{Droplet take-off}

Two scenarios are possible when a voltage higher than the take-off threshold is applied between the upper electrode and the gold micro-catenary from which the droplet acquires its electrical charge [21,22]. The droplet behavior depends on whether or not the departure is in competition with electrowetting. In the case of a competition with electrowetting the droplet remains stuck to the lower EWOD based chip. In the other case, the droplet takes-off as described in Section 3. Therefore, among several droplets, the droplets to be lifted can be selected in the open system thereby enabling the upper plate to be used as a storage memory [18] (Fig. 9).

Once on the upper plate, the droplet is flattened by the electrical force (see Section 4.2). The microstructures designed to prevent the droplet from sliding sideways enable a perfect control of the droplet cover location. Afterwards, droplets can be removed from the upper plate by gravity when the voltage between the micro-catenary and upper electrode is set to zero. However, usually it is necessary to reverse the take-off voltage in order to overcome the partial wetting of the droplet to the cover. 


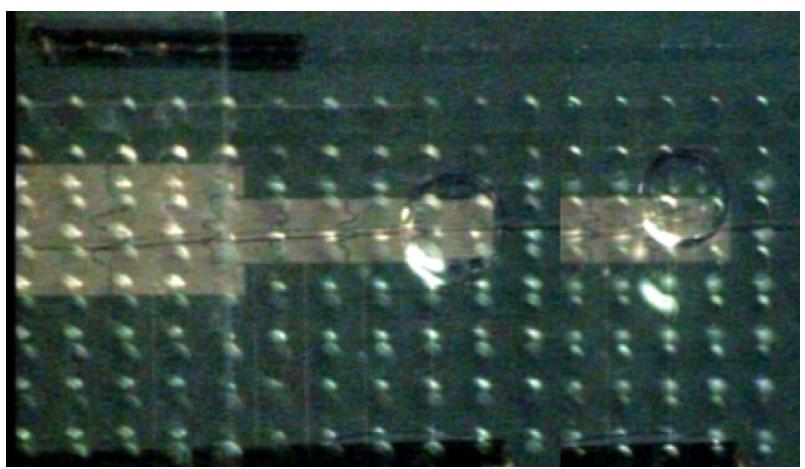

Fig. 9. Demonstration of the vertical displacement in the device. A deionized water droplet moves up from the chip array (left) to the top glass plate (right). The cylinders made in the dry film of Ordyl to prevent the droplet from sliding on the top glass plate are visible on the pictures.

The device described here could realize droplet dispensing, EWOD displacement on a first plane surface and reversible transfer to a second plane surface.

\subsection{Discussion}

In the present device, a voltage of about $600 \mathrm{~V}$ was used to lift droplets. It gives an electrical field value of about $3 \mathrm{kV} / \mathrm{cm}$ which is 2.5 times higher than the threshold value suggested by the result plotted in Fig. 1. Even if the voltage of $600 \mathrm{~V}$ is not a threshold, it should be recognized that the take-off voltage in the present device is superior to the results plotted in Fig. 1. On one hand, the wetting property of the chip surface (Teflon-like layer) is not as much hydrophobic as the experimental set-up electrode (carbon nanotubes). Furthermore, the wetting was forced by the EWOD based displacement and so the de-wetting process is at work before take-off. Here, the electrical force must overcome not only the weight of the droplet but also the capillary force. This last force is very difficult to predict and further work is necessary to take it into account. On the other hand, in our device, the lower electrode of the experimental set-up is replaced by a micro-catenary and a row of insulated electrodes. However the take-off mechanism remains the same and the physical considerations of Section 2 gives a good order of magnitude for the take-off voltage.

\section{Conclusion}

A uniform electric field exerts a lifting force on a conductive droplet immersed in a dielectric fluid and in contact with an electrode. If this electrode is the lower plate of a condenser, such a droplet bounces continuously and regularly between the two plates in an uncontrolled way. Back and forth motion can be controlled by coating the upper electrode with a dielectric layer and by adjusting the electric field direction.

The displacements studied experimentally and theoretically were integrated in a microfluidic device coupled to an electrowetting-based chip. The resulting device, demonstrating the handling of droplets in the 3D space, introduces a new generation of electrowetting-based microfluidic system.

\section{Acknowledgements}

We would like to thank Raymond Charles and Claude Chabrol for helping out with the experimental devices and set-up; Marc Plissonnier for providing us carbon nanotubes covered electrodes; Gaël Castellan, Olivier Constantin, Régis Blanc and Dominique Lauro for manufacturing the micro-components and finally, Nelly Bonifaci (CNRS Electrostatic and Dielectric Environments Laboratory) for the permittivity and conductivity measurements made on the mineral oil used in our experiments. This research is supported by the French Research Ministry on the Recherche Technologique de Base program (RTB).

\section{References}

[1] Y. Fouillet, J.-L. Achard, Microfluidique discrète et biotechnologie, Compte Rendu de l'Académie des Sciences 5 (2004) 577-588.

[2] Y. Fouillet, H. Jeanson, I. Chartier, A. Buguin, P. Silberzan, Déplacement de gouttes sur un microcaténaire, in: Proceedings of the SHF Microfluidique, Toulouse, France, 2002.

[3] P. Kolar, R.B. Fair, Non-contact electrostatic stamping for DNA microarray synthesis (poster), in: Proceedings of the SmallTalk2001, San Diego, USA, 2001.

[4] A. Khayari, A.T. Pérez, F.J. Garcia, A. Castellanos, Dynamics and deformation of a drop in a DC electric field, in: Proceedings of the IEEE Electrical Insulation and Dielectric Phenomena, Albuquerque, USA, 2003, pp. 682-685.

[5] N.N. Lebedev, I.P. Skal'skaya, Force acting on a conducting sphere in the field of a parallel plate condenser, Soviet Phys. Tech. Phys. 7 (1962) 268-270.

[6] T.B. Jones, Electromechanics of Particles, Cambridge University Press, 1995, pp. 183-185 (Chapter 7).

[7] K. Takahashi, H. Kajihara, M. Urago, S. Saito, Y. Mochimaru, T. Onzawa, Voltage required to detach an adhered particule by Coulomb interaction for micromanipulation, J. Appl. Phys. 90 (2001) 432-437.

[8] S. Saito, H. Himeno, K. Takahashi, Electrostatic detachment of an adhering particle from a micromanipulated probe, J. Appl. Phys. 93 (2003) 2219-2224

[9] K.K.S. Lau, J. Bico, K.B.K. Teo, M. Chhowalla, G.A.J. Amaratunga, W.I. Milne, G.H. McKinley, K.K. Gleason, Superhydrophobic carbon nanotube forests, Nano Lett. 3 (2003) 1701-1705.

[10] M.H. Davis, Two charged spherical conductors in a uniform electric field: forces and field strength, Q. J. Mech. Appl. Math. 17 (1964) 499511.

[11] R. Tobazéon, Behaviour of spherical and cylindrical particles in an insulating liquid subjected to a DC uniform field, in: Proceedings 11th ICDL, 1993, pp. 415-420.

[12] J. Vykoukal, J.A. Schwartz, F. Becker, P.R.C. Gascoyne, A programmable dielectrophoretic fluid processor for droplet-based chemistry, in: Proceedings of the Micro Total Analysis Systems 2001, Monterey, USA, 2001, pp. 72-74.

[13] T.B. Jones, M. Gunji, M. Washizu, M.J. Feldman, Dielectrophoretic liquid actuation and nanodroplet formation, J. Appl. Phys. 89 (2001) 14411448 .

[14] O.D. Velev, B.G. Prevo, K.H. Bhatt, On-chip manipulation of free droplets, Nature 426 (2003) 515.

[15] S. Nilson, S. Santesson, E. Degerman, T. Johansson, T. Laurell, J. Nilsson, J. Joyansson, Airborne chemistry for biological micro analysis, in: Proceedings of the Micro Total Analysis Systems 2000, Enschedel, the Netherlands, 2000, pp. 19-23.

[16] T.S. Sammarco, M.A. Burns, Heat-transfer analysis of microfabricated thermocapillary pumping and reaction devices, J. Micromech. Microeng. 10 (2000) 42-55. 
[17] B. Berge, Electrocapillarite et mouillage de films isolants par l'eau, Compte Rendu de l'Académie des Sciences 317 (1993) 157-163.

[18] Y. Fouillet, D. Jary, A.G. Brachet, C. Chabrol, J. Boutet, P. Clementz, D. Lauro, R. Charles, C. Peponnet, Design and validation of a complex generic fluidic microprocessor based on EWOD droplet for biological applications, in: Proceedings of the Micro Total Analysis Systems 2005, Boston, USA 2005 , p. 58.

[19] S.K. Cho, H. Moon, C.-J. Kim, Creating, transporting, cutting, and merging liquid droplets by electrowetting-based actuation for digital microfluidic circuits, J. Microelectromech. Syst. 12 (2003) 7080.

[20] R.B. Fair, V. Srinivasan, H. Ren, P. Paik, V.K. Pamula, M.G. Pollack, Electrowetting-based on-chip sample processing for integrated microfluidics, in: Proceedings of the IEEE Inter. Electron Devices Meeting, Washington, DC, USA, 2003, pp. 32.5.1-32.5.4.

[21] J.-M. Roux, Y. Fouillet, J.-L. Achard, Handling droplets in 3 dimensions for lab-on-chip applications, in: Proceedings of the Micro Total Analysis Systems 2004, Malmö, Sweden, 2004, pp. 581-583.

[22] J.-M. Roux, Y. Fouillet, J.-L. Achard, Déplacement 3D d'une microgoutte par des forces électrostatiques pour des laboratoires sur puce, in: Proceedings of the SHF Microfluidique, Toulouse, France, 2004.

\section{Biographies}

Jean-Maxime Roux received his engineering diploma from the Institut National Polytechnique de Grenoble (INPG) in 2003. He is currently studying for a $\mathrm{PhD}$ in the Department of Technology for Biology and Health at the CEA/LETI in Grenoble. His current research interests are electrohydrodynamic and droplet handling for lab-on-chip applications.

Yves Fouillet received his "Agrégation de mécanique" in 1987 and a PhD degree from the Institut National Polytechnique de Grenoble (INPG), France, in 1992 (Numerical simulation of shear flow). In 1992 he joined the CEA-LETI for MEMS development. Since 1998, he has worked on microfluidic. His current research interests include lab on a chip, droplet handling and electrowetting.

Jean-Luc Achard is presently head of the microfluidic group of the Laboratoire des Ecoulements Géophysiques et Industriels (LEGI) located at Grenoble. His current research interests include droplet handling in miniaturized systems for biofluidic applications. He received his DSc from the Institut National Polytechnique de Grenoble (INPG), France, in 1978. He began his career studying thermal hydraulics instabilities occurring in nuclear reactor plants. Then he has specialized in modelling both dispersed two-phase flows and single inclusions dynamics. 